\title{
General Anesthesia With Dexmedetomidine and Remifentanil in a 3-Year-Old Child: An Alternative Anesthetic Regimen to Allay Parental Concerns of the Potential Neurocognitive Effects of General Anesthesia
}

\author{
Hina Walia ${ }^{\mathrm{a}, \mathrm{d}}$, Emmett Whitaker ${ }^{\mathrm{a}, \mathrm{b}}$, Gregory Pearson ${ }^{\mathrm{c}}$, Joseph D. Tobias ${ }^{\mathrm{a}}$
}

\begin{abstract}
Recent attention has been focused on the potential neurocognitive effects of general anesthesia during infancy and early life. Although the exact implications of this effect have yet to be proven, media attention has been raised and parents may be aware of such problems, thereby questioning the safety of general anesthesia. Although spinal and regional anesthetic techniques have seen an increased use in an attempt to eliminate the concerns of such problems, many surgical procedures may not be amenable to regional anesthesia. We present a 3-year-old boy who presented for excision of an enlarging cyst on his left ear. During the preoperative visit with the surgeon, the patient's mother voiced concerns regarding the potential neurocognitive effects of general anesthesia. General anesthesia was provided using a combination of dexmedetomidine and remifentanil. Previous reports of the use of this unique combination of agents are reviewed and its role in this scenario was discussed.
\end{abstract}

Keywords: Dexmedetomidine; Remifentanil; Pediatric anesthesia; Neurotoxicity

\section{Introduction}

Recent attention has been focused on the potential deleterious neurocognitive effects of general anesthesia during infancy and early life [1-6]. Although the exact implications of this ef-

Manuscript accepted for publication February 19, 2016

aDepartment of Anesthesiology \& Pain Medicine, Nationwide Children's Hospital, Columbus, OH, USA

${ }^{b}$ Department of Anesthesiology \& Pain Medicine, The Ohio State University College of Medicine, Columbus, OH, USA

'Department of Plastic \& Reconstructive Surgery, Nationwide Children's Hospital and The Ohio State University College of Medicine, Columbus, $\mathrm{OH}$, USA

${ }^{\mathrm{d} C}$ Corresponding Author: Hina Walia, Department of Anesthesiology \& Pain Medicine, Nationwide Children's Hospital, 700 Children's Drive, Columbus, OH 43205, USA. Email: Hina.Walia@Nationwidechildrens.org

doi: http://dx.doi.org/10.14740/jmc2453w fect have yet to be proven, media attention has been raised and many parents are aware of the potential for such problems [7, 8]. The potential neurocognitive effects of various general anesthetic agents have been demonstrated in laboratory animals and suggested from retrospective clinical trials [1-6]. The anesthetic agents identified as possible neurotoxins include either $\gamma$-amino-butyric-acid (GABA) agonists including the volatile anesthetic agents, benzodiazepines, barbiturates and propofol or N-methyl-D-aspartate (NMDA) antagonists such as nitrous oxide or ketamine. Although spinal and regional anesthesia are acceptable alternatives and have seen increased use in an attempt to eliminate the concerns of such problems, many surgical procedures may not be amenable to regional anesthesia [9, 10].

We report a 3-year-old boy presenting for excision of an enlarging left ear cyst. During the preoperative visit with the surgeon, the patient's mother expressed concerns regarding the potential neurocognitive effects of general anesthesia. General anesthesia was provided using a combination of dexmedetomidine and remifentanil. Previous reports of the use of this unique combination of agents are reviewed and its potential role in this scenario was discussed.

\section{Case Report}

Institutional Review Board approval is not required for presentation of single case reports at Nationwide Children's Hospital (Columbus, OH). The patient was a 3-year-old, $14 \mathrm{~kg}$ boy who presented for excision of a left ear cyst that had been enlarging over the past 11 months. His past medical history included varicella, parainfluenza respiratory infection, hand-foot-mouth disease, and a single episode of a febrile seizure at 17 months of age. There was no past surgical history. The patient had no ongoing co-morbid conditions and he was not taking any regularly scheduled medications. Preoperative physical examination revealed a young child in no acute distress. Preoperative laboratory evaluation was normal. The patient was admitted on the day of his surgery. During the preoperative surgical visit, the mother had expressed concerns regarding the potential neurocognitive effects of general anesthesia. These concerns were based on information from various internet sites and the media. Based on these concerns, it was decided 
to proceed with a general anesthesia using dexmedetomidine and remifentanil. Two options were presented for obtaining intravenous (IV) access including either a brief exposure to sevoflurane and nitrous oxide or a topical anesthetic cream with awake IV cannulation. The mother chose brief exposure to sevoflurane; however, premedication with oral midazolam was declined. The patient was held nil per os for solids for $6 \mathrm{~h}$ and for clear liquids for $4 \mathrm{~h}$ prior to surgery. The patient was transported to the operating room and routine American Society of Anesthesiologists' monitors were placed. Preoperative hemodynamic data included blood pressure (BP) $90 / 58 \mathrm{~mm}$ $\mathrm{Hg}$ and heart rate (HR) 97 beats/min. Seventy percent nitrous oxide in oxygen was administered via face mask for $60 \mathrm{~s}$ followed by the administration of $8 \%$ sevoflurane. Peripheral IV cannulation was achieved within 2 min and the nitrous oxide and sevoflurane were discontinued. Dexmedetomidine $(1 \mu \mathrm{g} /$ $\mathrm{kg})$ and fentanyl $(1.5 \mu \mathrm{g} / \mathrm{kg})$ were administered intravenously over 1 - 2 min as a bolus dose and a laryngeal mask airway (LMA) was placed. Dexmedetomidine and remifentanil infusions were started at $1 \mu \mathrm{g} / \mathrm{kg} / \mathrm{h}$ and $0.2 \mu \mathrm{g} / \mathrm{kg} / \mathrm{min}$, respectively. To ensure an adequate depth of anesthesia, a bispectral index (BIS) monitor was placed. Due to technical problems, only intermittent readings were able to be obtained, ranging from 40 to 60 . Sensory nerve supply to the ear was blocked by local subcutaneous infiltration with $0.25 \%$ bupivacaine with epinephrine $(1: 200,000)$. Spontaneous ventilation was maintained throughout the case with an inspired oxygen concentration of $40-50 \%$. Intraoperatively, the HR varied from 90 to 130 beats/min. The BP was well maintained during the intraoperative course (BP: 70 - 100/40 - $50 \mathrm{~mm} \mathrm{Hg}$ ). No abrupt changes in oxygen saturation or end-tidal carbon dioxide were noted. The remainder of the intraoperative course was unremarkable. The surgical procedure lasted approximately $70 \mathrm{~min}$. At the conclusion of the procedure, the dexmedetomidine and remifentanil infusions were discontinued. The wake-up time from the completion of the surgical procedure to removal of the LMA was $1-2$ min. The patient was transported to the post-anesthesia care unit (PACU). The remainder of his postoperative course was uncomplicated and he was discharged home on the same day.

\section{Discussion}

Dexmedetomidine is an $\alpha_{2}$-adrenergic agonist which initially received approval by the United States Food \& Drug Administration (FDA) in 1999 for the sedation of adults during mechanical ventilation. In 2009, it subsequently received FDA approval for monitored anesthesia care (MAC). Although only FDA approved for use in adults, dexmedetomidine has been shown to be efficacious in several different clinical scenarios in infants and children including sedation during mechanical ventilation, procedural sedation, supplementation of postoperative analgesia, prevention of emergence delirium, and treatment of withdrawal [11]. However, to date, there are limited reports of its use as the primary part of a general anesthetic regimen [12-14].

Beneficial and protective effects on the central nervous system during hypoxia or ischemia have been demonstrated with dexmedetomidine in animal studies [15-19]. Unlike agents that act as GABA agonists or NMDA antagonists, animal data have demonstrated limited impact on apoptosis and neurocognitive outcome $[20,21]$. In addition to its lack of proapoptotic effect, dexmedetomidine has been shown to decrease the pro-apoptotic effect of the volatile anesthetic agent, isoflurane, in laboratory animals $[20,21]$.

Remifentanil is a synthetic opioid derivative that was introduced into clinical practice in the United States in 1996 [22]. It has a strong affinity for $\mu$ opioid receptors with less affinity for other opioid receptors ( $\kappa$ and $\sigma$ ). Its potency and respiratory depressant effect is generally considered to be twice that of fentanyl. However, its half-life is significantly shorter due to modification of its chemical structure with the incorporation of a methyl-ester ring into the molecule thereby allowing its hydrolysis by non-specific plasma and tissue esterases. These characteristics result in a unique pharmacokinetic profile with a rapid onset, easy titration by continuous infusion, and a short context-sensitive half-life with rapid elimination across all age ranges, including neonates. These characteristics make it a valuable agent for the provision of intense intraoperative analgesia with a rapid recovery regardless of the dose or duration of the infusion. Furthermore, the opioids have been shown in laboratory animals to have limited pro-apoptotic effects [2-5]. In particular, remifentanil has been shown to have neuroprotective effects during ischemia in laboratory animals and potentially the ability to blunt the pro-apoptotic effects of other agents [23-25].

In our patient, the mother had requested the use of "neuro-safe" medications given her concerns following her reading of articles from the internet and publications from the lay press. As such, we chose a combination of dexmedetomidine and remifentanil, two medications which have been shown in laboratory animals to be free of pro-apoptotic effects. When choosing this anesthetic, several concerns were encountered including whether premedication with midazolam should be used given concerns regarding benzodiazepines and their agonism at GABA receptors. Although our common practice includes the use of oral midazolam for anxiolysis to prevent separation anxiety, alternatives include parental presence or the use of other distraction techniques to avoid the need for such medications [24, 26]. Alternatively, although onset times may be longer than oral midazolam, both oral and intranasal dexmedetomidine have been used for premedication prior to anesthetic induction.

Another aspect of concern was the technique for IV cannulation as this is usually performed following the induction of general anesthesia with sevoflurane in pediatric patients. After discussion with the mother, it was decided to proceed without premedication, but to briefly administer nitrous oxide with sevoflurane to allow for placement of an intravenous cannula. Alternatively, use of a topical anesthetic cream may provide an alternative for awake venous cannulation without the need for sevoflurane or nitrous oxide. The presence of an IV cannula would also allow the preoperative administration of IV dexmedetomidine which may be used to facilitate parental separation and transport to the operating room.

Following placement of the IV cannula, the sevoflurane was discontinued and a bolus dose of fentanyl and dexmedeto- 
Table 1. Previous Reports of Dexmedetomidine-Remifentanil for General Anesthesia

\begin{tabular}{|c|c|c|}
\hline Authors and reference number & Patient demographics & Intraoperative care \\
\hline Moharir and Tobias [14] & $\begin{array}{l}\text { An 11-year-old patient } \\
\text { with Duchenne muscular } \\
\text { dystrophy for cardiac } \\
\text { catheterization }\end{array}$ & $\begin{array}{l}\text { Dexmedetomidine and remifentanil were started at } 0.7 \mu \mathrm{g} / \mathrm{kg} / \mathrm{h} \text { and } 0.1 \mu \mathrm{g} / \mathrm{kg} / \\
\text { min, respectively without bolus dosing. After ensuring an adequate depth of } \\
\text { sedation (approximately } 10 \mathrm{~min} \text { ), the groin was infiltrated with } 1 \% \text { lidocaine } \\
\text { prior to cannula placement. The procedure was completed in } 45 \mathrm{~min} \text {. The } \\
\text { dexmedetomidine and remifentanil infusions were discontinued. The patient } \\
\text { was awake and conversing within } 5 \text { min. The post-procedure course was } \\
\text { unremarkable. }\end{array}$ \\
\hline
\end{tabular}

midine was administered followed by placement of an LMA. Maintenance anesthesia was provided by continuous infusions of dexmedetomidine and remifentanil, which were started at $1.0 \mu \mathrm{g} / \mathrm{kg} / \mathrm{h}$ and $0.2 \mu \mathrm{g} / \mathrm{kg} / \mathrm{min}$, respectively and titrated according to clinical need. Given its intense analgesic effects, the remifentanil infusion was titrated to control the hemodynamic response to surgical stimulation and allow for spontaneous ventilation, while the dexmedetomidine infusion was used to provide amnesia and adjusted according to the BIS. Previous reports regarding the use of a combination of dexmedetomidine and remifentanil for general anesthesia are summarized in Table $1[13,14,27]$.

While anecdotal experience suggests efficacy of a combination of dexmedetomidine and remifentanil for intraoperative anesthetic care, potential concerns include lack of significant data demonstrating the amnestic effects of dexmedetomidine, adverse hemodynamic effects, and the potential for prolonged awakening given the longer duration of action of dexmedetomidine compared to other agents. Ensuring adequate amnesia is one of the goals of intraoperative anesthetic care. Although it is accepted as an effective agent for providing sedation and anxiolysis, there are limited data to clearly demonstrate the amnestic effects of dexmedetomidine. In a randomized, cross-over trial, nine adults received either propofol or dexmedetomidine using computer-controlled infusions targeting an effect-site concentration of 1,2 , and $4 \mu \mathrm{g} / \mathrm{mL}$ for propofol or a plasma concentration of $0.6,1.2$, and $2.4 \mathrm{ng} / \mathrm{mL}$ for dexmedetomidine [28]. Using these graded infusions, the authors then compared the BIS number with the depth of sedation as assessed using the observer's assessment of alertness and sedation (OAA/S) score. The cutoff values of BIS for an OAA/S score $\leq 2$ (a depth of sedation that would ensure amnesia and prevent recall) were obtained by analysis of receiver operating characteristic curves. The median BIS values at OAA/S scores of 1, 2, 3, 4, and 5 during propofol sedation were 95.5,
$78,67,57$, and 34, respectively. Median BIS values at OAA/S scores of $1,2,3,4$, and 5 during dexmedetomidine sedation were $95,62,45.5,39.5$, and 24.5 , respectively. BIS values were significantly less with dexmedetomidine than propofol at OAA/S responsiveness scores of 2, 3, and 4. With propofol, the calculated cutoff BIS values for an OAA/S score $\leq 2$ were 67 (sensitivity of $86 \%$, specificity of $97 \%$, and area under the curve of 0.98 ). With dexmedetomidine, the values were lower: 46 (sensitivity of $84 \%$, specificity of $91 \%$, and area under the curve of 0.96). Other studies have demonstrated effective amnesia as evidenced by impaired long-term picture recall with dexmedetomidine [29, 30].

Regardless of the anesthetic agents used, adverse effects on respiratory and hemodynamic function may occur. Like all opioids, remifentanil will depress respiratory function in a dose-related manner resulting in respiratory depression and eventually apnea. Higher dosing regimens may require assisted or controlled ventilation. However, when compared to other anesthetic agents such as propofol, there is less impairment of respiratory function with dexmedetomidine [28, 31]. Adverse hemodynamic effects, albeit rare in the pediatric-aged patient, include bradycardia and hypotension. Hemodynamic effects are more common following bolus dosing, in patients with comorbid cardiac disease, and generally resolve with a decrease of the infusion rate [11].

In summary, we present the use of a combination of dexmedetomidine and remifentanil to provide anesthesia during a minor surgical procedure requiring general anesthesia. The impetus behind such care was prompted by maternal concerns over the potential long-term deleterious effects of general anesthesia on neurocognitive outcomes. Although there remains limited anecdotal experience with such a combination, given the animal data demonstrating a lack of a pro-apoptotic effect, this potentially "neurocognitive safe" anesthetic may warrant further investigation. 


\section{References}

1. Sun L. Early childhood general anaesthesia exposure and neurocognitive development. $\mathrm{Br} \mathrm{J}$ Anaesth. 2010;105(Suppl 1):i61-68.

2. Blaylock M, Engelhardt T, Bissonnette B. Fundamentals of neuronal apoptosis relevant to pediatric anesthesia. Paediatr Anaesth. 2010;20(5):383-395.

3. Mellon RD, Simone AF, Rappaport BA. Use of anesthetic agents in neonates and young children. Anesth Analg. 2007;104(3):509-520.

4. Loepke AW, Soriano SG. An assessment of the effects of general anesthetics on developing brain structure and neurocognitive function. Anesth Analg. 2008;106(6):16811707.

5. Istaphanous GK, Loepke AW. General anesthetics and the developing brain. Curr Opin Anaesthesiol. 2009;22(3):368373.

6. Jevtovic-Todorovic V, Hartman RE, Izumi Y, Benshoff ND, Dikranian K, Zorumski CF, Olney JW, et al. Early exposure to common anesthetic agents causes widespread neurodegeneration in the developing rat brain and persistent learning deficits. J Neurosci. 2003;23(3):876-882.

7. Weiss M, Bissonnette B, Engelhardt T, Soriano S. Anesthetists rather than anesthetics are the threat to baby brains. Paediatr Anaesth. 2013;23(10):881-882.

8. Davidson AJ. Anesthesia and neurotoxicity to the developing brain: the clinical relevance. Paediatr Anaesth. 2011;21(7):716-721.

9. Davidson AJ, Morton NS, Arnup SJ, de Graaff JC, Disma N, Withington DE, Frawley G, et al. Apnea after Awake Regional and General Anesthesia in Infants: The General Anesthesia Compared to Spinal Anesthesia Study--Comparing Apnea and Neurodevelopmental Outcomes, a Randomized Controlled Trial. Anesthesiology. 2015;123(1):38-54.

10. Davidson AJ, Disma N, de Graaff JC, Withington DE, Dorris L, Bell G, Stargatt R, et al. Neurodevelopmental outcome at 2 years of age after general anaesthesia and awake-regional anaesthesia in infancy (GAS): an international multicentre, randomised controlled trial. Lancet. 2016;387(10015):239-250.

11. Tobias JD. Dexmedetomidine: applications in pediatric critical care and pediatric anesthesiology. Pediatr Crit Care Med. 2007;8(2):115-131.

12. Dewhirst E, Naguib A, Tobias JD. Dexmedetomidine as part of balanced anesthesia care in children with malignant hyperthermia risk and egg allergy. J Pediatr Pharmacol Ther. 2011;16(2):113-117.

13. Burnett B, Schwartz L. Tobias J. Anaesthesia with dexmedetomidine and remifentanil in a child with mitochondrial myopathy. South Afr J Anaesth Analg. 2011;17:262264.

14. Moharir A, Tobias JD. Monitored anesthesia care with dexmedetomidine and remifentanil during cardiac catheterization in a patient with Duchenne muscular dystrophy and malignant hyperthermia susceptibility. Anaesth Pain Intensive Care 2013;17:292-295.
15. Hoffman WE, Kochs E, Werner C, Thomas C, Albrecht RF. Dexmedetomidine improves neurologic outcome from incomplete ischemia in the rat. Reversal by the alpha 2-adrenergic antagonist atipamezole. Anesthesiology. 1991;75(2):328-332.

16. Kuhmonen J, Pokorny J, Miettinen R, Haapalinna A, Jolkkonen J, Riekkinen P, Sr., Sivenius J. Neuroprotective effects of dexmedetomidine in the gerbil hippocampus after transient global ischemia. Anesthesiology. 1997;87(2):371-377.

17. Kuhmonen J, Haapalinna A, Sivenius J. Effects of dexmedetomidine after transient and permanent occlusion of the middle cerebral artery in the rat. J Neural Transm (Vienna). 2001;108(3):261-271.

18. Sifringer M, von Haefen C, Krain M, Paeschke N, Bendix I, Buhrer C, Spies CD, et al. Neuroprotective effect of dexmedetomidine on hyperoxia-induced toxicity in the neonatal rat brain. Oxid Med Cell Longev. 2015;2015:530371.

19. Ma D, Hossain M, Rajakumaraswamy N, Arshad M, Sanders RD, Franks NP, Maze M. Dexmedetomidine produces its neuroprotective effect via the alpha 2A-adrenoceptor subtype. Eur J Pharmacol. 2004;502(1-2):87-97.

20. Sanders RD, Sun P, Patel S, Li M, Maze M, Ma D. Dexmedetomidine provides cortical neuroprotection: impact on anaesthetic-induced neuroapoptosis in the rat developing brain. Acta Anaesthesiol Scand. 2010;54(6):710-716.

21. Sanders RD, Xu J, Shu Y, Januszewski A, Halder S, Fidalgo A, Sun P, et al. Dexmedetomidine attenuates isoflurane-induced neurocognitive impairment in neonatal rats. Anesthesiology. 2009;110(5):1077-1085.

22. Marsh DF, Hodkinson B. Remifentanil in paediatric anaesthetic practice. Anaesthesia. 2009;64(3):301-308.

23. Park SW, Yi JW, Kim YM, Kang JM, Kim DO, Shin MS, Kim CJ, et al. Remifentanil alleviates transient cerebral ischemia-induced memory impairment through suppression of apoptotic neuronal cell death in gerbils. Korean $\mathrm{J}$ Anesthesiol. 2011;61(1):63-68.

24. Tourrel F, de Lendeu PK, Abily-Donval L, Chollat C, Marret S, Dufrasne F, Compagnon P, et al. The antiapoptotic effect of remifentanil on the immature mouse brain: an ex vivo study. Anesth Analg. 2014;118(5):1041-1051.

25. Fodale V, Schifilliti D, Pratico C, Santamaria LB. Remifentanil and the brain. Acta Anaesthesiol Scand. 2008;52(3):319-326.

26. Kain ZN. Premedication and parental presence revisited. Curr Opin Anaesthesiol. 2001;14(3):331-337.

27. Suleman MI, Edala T, Abraham E, Siddiqu MS. NonTrigger Anesthesia Management in a Patient With Leigh's Syndrome Presenting for Dental Rehabilitation. Anesth Pain Med. 2015;5(6):e28804.

28. Kasuya Y, Govinda R, Rauch S, Mascha EJ, Sessler DI, Turan A. The correlation between bispectral index and observational sedation scale in volunteers sedated with dexmedetomidine and propofol. Anesth Analg. 2009;109(6):1811-1815.

29. Hayama HR, Drumheller KM, Mastromonaco M, Reist C, Cahill LF, Alkire MT. Event-related functional magnetic resonance imaging of a low dose of dexmedeto- 
midine that impairs long-term memory. Anesthesiology. 2012;117(5):981-995.

30. Eremenko AA, Chernova EV. [Dexmedetomidine use for intravenous sedation and delirium treatment during early postoperative period in cardio-surgical patients]. Anesteziol Reanimatol. 2013;5:4-8.
31. Mahmoud M, Jung D, Salisbury S, McAuliffe J, Gunter J, Patio M, Donnelly LF, et al. Effect of increasing depth of dexmedetomidine and propofol anesthesia on upper airway morphology in children and adolescents with obstructive sleep apnea. J Clin Anesth. 2013;25(7):529541. 